\title{
Modeling and simulation of Constant Phase Element for battery Electrochemical Impedance Spectroscopy
}

\author{
Edoardo Locorotondo, Luca Pugi, Lorenzo Berzi, \\ Marco Pierini \\ Dept. of Industrial Engineering \\ University of Florence \\ Florence, Italy \\ Corresponding author: Edoardo.locorotondo@unifi.it
}

\author{
Santo Scavuzzo, Alessandro Ferraris, Andrea \\ Giancarlo Airale, Massimiliana Carello \\ Dept. of Mechanical and Aerospace Engineering \\ Politecnico di Torino \\ Turin, Italy
}

\begin{abstract}
This paper presents a set of time-domain electrical equivalent circuit models for battery voltage prediction under arbitrary current profiles. The circuit model is composed by passive electrical components like resistance, inductance and capacitance. The constant phase elements are introduced in the model, which are usually used in electrochemical impedance spectroscopy analysis to describe the electrical property of double layer capacitors between electrode and electrolyte. The aim of the paper is the modeling in the time-domain of the constant phase elements. Two models are proposed. Model's accuracy and run speed are evaluated by comparing experimental results obtained by an appropriate testbed and simulation results obtained by the implementation and the execution of the model using Matlab software.
\end{abstract}

Keywords-Constant Phase Elements; Electrochemical Impedance Spectroscopy; Lithium batteries; Electrical Circuit Models

\section{INTRODUCTION}

Electrochemical energy accumulators, mainly Lithium Batteries (LIBs), are becoming more and more important in automotive industry and in smart city development [1]. Nowadays, LIBs are considered as the main energy storage for Electrical Vehicles (EVs). In order to increase battery performance in terms of delivering/absorbing electrical power and to ensuring high safety levels, it is necessary to predict some parameters, which cannot be directly measured, as State Of Charge (SOC) [2], State Of Health (SOH) [3], internal impedance [4] and internal temperature [5]. Since battery voltage, current and surrounding temperature can be measured by the Battery Management System (BMS), a mathematical model is required in order to estimate the parameters cited previously. Modeling and management of LIBs are topics more and more discussed in European research programs (Horizon 2020). More accurate battery model should help BMS to increase not only the performance of the battery system but of the entire EV. Indeed more control strategy in Electrical Control Unit (ECU) of the vehicle requires the estimation of battery internal states, as shown in [6]. In literature exist many methodologies for battery modeling and battery state

This work is part of the OBELICS project which has received funding from the European Union Horizon 2020 research and innovation program under grant agreement No. 769506. estimation [7], [8]. The focus of this work is on battery impedance modeling, using Electrical Equivalent Circuit (EEC) models. The dependence of battery impedance parameters on the SOC, SOH [7] and on the internal temperature [9] has encouraged us to analyze the impedance, considering it as the basis for the battery state estimation. There are two main types of EEC impedance model: one developed using a series/parallel configuration of resistance, capacitance and inductance (lumped system), included in the Thevenin equivalent circuit model [2]-[4]; another one developed using the so-called distributed parameters [10], which transfer functions are irrationals. The first one is identified and parametrized by step-response method, assuming that the system is working in an appropriate operating condition [4]. However, using this method, impedance estimations are strongly limited because they do not represent the wealth of information inherently present in broadband battery electrical impedance. Compared to the step-response methods, the Electrochemical Impedance Spectroscopy (EIS) measurements use swept frequency sinusoidal signal excitation allowing the direct measurement of the system response in broadband and any working point. In this case, the impedance measurements are usually plotted in Nyquist, otherwise in Bode plots. Therefore, impedance circuit parameters are estimated using appropriate best fitting algorithms in order to simulate the same impedance measurements in Nyquist plot [11]. As shown in [10]-[13], distributed parameters like the Constant Phase Elements (CPEs) are introduced to accurately fitting the battery impedance curve in Nyquist. These parameters have constant phase in all the frequency band and they are represented with irrational function. Aim of this work is the realization of a time-domain model of one CPE and many CPEs in series/parallel configurations, which define the EEC battery model. This model will be implemented in Matlab software, in order to simulate the evolution of the CPE and EEC model's voltage response in time-domain, comparing it with experimental data. This paper is organized as follows. Section II briefly introduces battery modeling in EIS, introducing the distributed parameters, in particular the CPE; Section III and IV describes the idea of the time-domain 
model of CPE and battery impedance; Section V evaluates the results of battery voltage response reproduced by EEC model implemented, analyzing the model accuracy, stability and calculation time.

\section{CONSTANT PHASE ELEMENT}

\section{A. Analysis in frequency-domain}

The Constant Phase Element (CPE) is a particular equivalent circuit passive element, which improves the match between the fit and the experimental battery EIS measurement in broadband. Usually it is used to describe the electrical properties of double layer capacitors [13] between the battery electrode and the electrolyte [14]. Its transfer function in frequency domain is defined as follows:

$$
Z_{C P E}(s)=\frac{1}{s^{\alpha} C}
$$

Where $\alpha \in[-1,1]$ is called depression factor of the CPE, $s=$ $j \omega$ is the complex Laplace variable, $\omega$ is the angular frequency. It's noticeable by (1) that the CPE should be represented as a non-ideal capacity (if $\alpha \in(0,1])$ or nonideal inductance (if $\alpha \in[-1,0)$ ). Indeed CPE can represent perfectly a capacitance $(\alpha=1)$, resistance $(\alpha=0)$ or an inductance $(\alpha=-1)$ parameter. The important property of the CPE in frequency-domain are shown by Nyquist plot in Fig. 1 and Fig. 2. In the circuit of Fig.1 (above) it's noticeable that the complex impedance of an ideal capacity component (CPE with $\alpha=1$ ) describes only a semicircumference with a radius equivalent to $R_{1} / 2$ and shifted of $R_{0}$ by the origin point. Using CPEs with variable $\alpha$ in the range $(0,1)$ it's possible to describe the Nyquist plot in variable semi-ellipse, which is common characteristic for impedance in the battery electrode/electrolyte. Other typical circuits configuration that describe the battery impedance in Nyquist plot at High and Low Frequencies (HF and LF) are respectively shown in Fig.1 (right) and Fig.2. Circuit of Fig.1 (right) describes this Nyquist plot if the capacitances of the two CPEs differ at least one order of magnitude (in this case if $C_{2}>>C_{1}$ ). In the Fig. 2 impedance is described by a semi-ellipse and a straight line of slope equivalent to $\alpha_{2} \pi / 2$. In particular, defining $\tau_{1}=R_{1} C_{1}, \tau_{2}=R_{1}\left(C_{1}+C_{2}\right)$ and $T=\tau_{2} / \tau_{1}$, impedance line become more blunt with increasing $T$ [15]. Last consideration is the following: usually in the battery the slope of the straight line at the LF is equal to $\alpha=0.5$. This particular CPE is the so-called Warburg impedance and this is usually used to describe the diffusion of lithium ions in the porous active material of the electrodes [14].

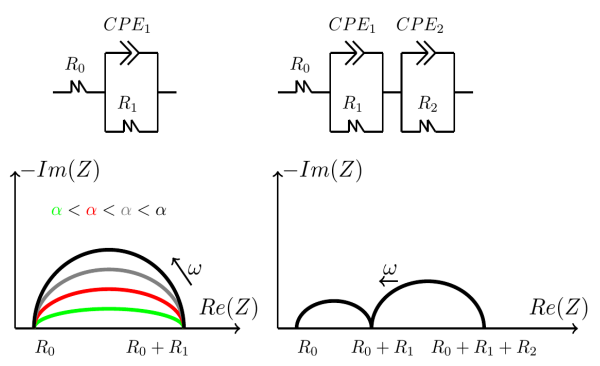

Fig. 1. Circuit $R_{0}-\left(R_{1} / / C P E_{1}\right)$ and its Nyquist diagram (left); Circuit $R_{0^{-}}$ $\left(R_{1} / / C P E_{1}\right)-\left(R_{2} / / C P E_{2}\right)$ and its Nyquist diagram (right).
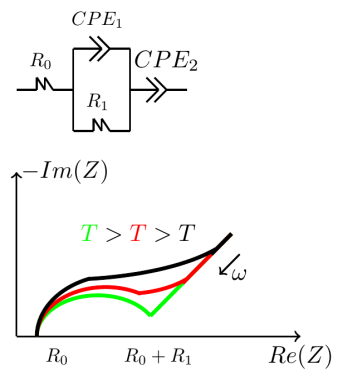

Fig. 2. Circuit $R_{0}-\left(R_{1} / / C P E_{1}\right)-C P E_{2}$ and its Nyquist diagram.

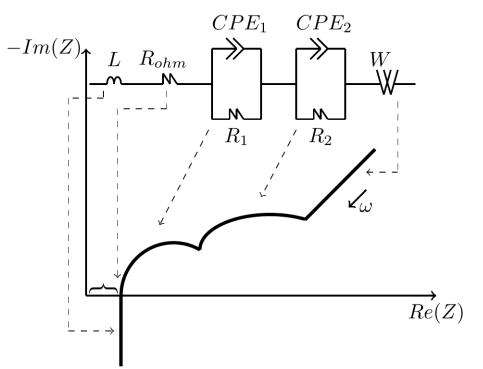

Fig. 3. Reference Nyquist diagram of Li-Ion cell and EIS modeling using Equivalent Electrical circuit with Constant Phase Elements $(C P E)$ and Warburg impedance ( $W$, is a CPE with $\alpha=0.5$ ).

\section{B. Battery Modeling in EIS}

LIBs usually show similar Nyquist plot as in Fig. 3. In particular EIS equivalent circuit in Fig. 3 proved to produce a relatively good fit to the measured EIS data in terms of stability, tolerance and relative error. This circuit can be subdivided in five sections, which separately describe different internal electrochemical processes of the LIB. It's noticeable that impedance circuit configuration in Fig. 3 is the series of the HF and LF circuit configurations shown in Fig.1 (right) and Fig. 2, adding an inductance component. Indeed, at highest frequency, LIB shows an inductive behavior due to the metal elements of the cell and cables [14]. In the case of EIS measurements, cell internal impedance is represented by distributed parameters like CPEs and Warburg impedances. In the next sections, the aim is the realization of time-domain model of this particular distributed parameter in order to simulate the short-time voltage response behaviors of battery.

\section{Modeling of the Constant Phase ElEment}

Starting from the transfer function (1), the analytical equation of CPE's voltage response is defined using different current inputs: step signals and continuous signals. The first step is to extract the Laplace Anti-Transform of $Z_{C P E}$ (1). Let's solve this by taking Laplace formula first:

$$
\mathcal{L}\left\{t^{n}\right\}=\frac{\Gamma[n+1]}{s^{n+1}}
$$

Where $\Gamma[n]=(n-1)$ ! is the Gamma function.

Substituting $\alpha=n+1$ and dividing all terms of Equation (2) with the capacitance value $C$, the Inverse Laplace Transform is the following:

$$
\mathcal{L}^{-1}\left\{\frac{1}{s^{\alpha} C}\right\}=\frac{t^{\alpha-1}}{\Gamma[\alpha] C}
$$




\section{A. Step Response}

The Laplace Transform of a current step $I_{\text {step }}(t)=I u[t]$ of amplitude $I$, can be expressed as:

$$
I_{\text {step }}(s)=\frac{I}{s}
$$

The CPE's voltage response $v_{C P E}(s)$ by an input current step (4), using (1), is the following:

$$
v_{C P E}(s)=Z_{C P E}(s) I_{\text {step }}(s)+\frac{v_{C P E, 0}}{s}=\frac{I}{s^{\alpha+1} C}+\frac{v_{C P E, 0}}{s}
$$

Using the inverse Laplace transform (3), the voltage response evolution $v_{C P E}(5)$ in the time-domain, applying an input current step, is represented by:

$$
v_{C P E}(t)=v_{C P E, 0}+\left(\frac{I t^{\alpha}}{\Gamma[\alpha+1] C}\right) u[t]
$$

\section{B. Square wave response}

In the case of single square wave current signal $I_{\text {square }}(t)=I\left(u\left[t-t_{1}\right]-u\left[t-t_{2}\right]\right)$ of start and final time $t_{1}$ and $t_{2}$ :

$$
v_{C P E}(s)=\frac{1}{s^{\alpha+1} C} I\left(e^{-s t_{2}}-e^{-s t_{1}}\right)+\frac{v_{C P E, 0}}{s}
$$

Where $I$ is the amplitude of current square wave signal. The inverse Laplace transform of Equation (7) is the following:

$$
\begin{aligned}
& v_{C P E}(t)=v_{C P E, 0}+ \\
& \frac{I}{\Gamma[\alpha+1] C}\left[\left(t-t_{1}\right)^{\alpha} u\left[t-t_{1}\right]-\left(t-t_{2}\right)^{\alpha} u\left[t-t_{2}\right]\right]
\end{aligned}
$$

Therefore, the voltage response evolution $v_{C P E}$ obtained by a current square wave excitation signal is represented by Equation (8).

\section{Trapezoidal Response}

The trapezoidal current signal $I_{\text {trapz }}(t)$ of start and final time $t_{1}$ and $t_{2}$ should be modelled in time-domain as a difference of two ramps signals as shown in the following formula:

$$
\begin{aligned}
& I_{\text {trapz }}(t)=\left(I_{1}+\frac{I_{2}-I_{1}}{T}\left(t-t_{1}\right)\right) u\left[t-t_{1}\right]- \\
& \left(I_{2}+\frac{I_{2}-I_{1}}{T}\left(t-t_{2}\right)\right) u\left[t-t_{2}\right]
\end{aligned}
$$

Where $I_{1}$ and $I_{2}$ are respectively the amplitudes of the trapezoidal signal at time $t_{1}$ and $t_{2}$ and $T=t_{2}-t_{1}$ is the time distance.

The CPE voltage response $v_{C P E}(s)$ by the trapezoidal signal (9) in frequency-domain is given by:

$$
\begin{aligned}
& v_{C P E}(s)=Z_{C P E}(s) I_{s t e p}(s)+\frac{v_{C P E, 0}}{s}= \\
& \frac{1}{s^{\alpha+1} C}\left[I_{1} e^{-s t_{1}}-I_{2} e^{-s t_{2}}\right]+\frac{I_{2}-I_{1}}{s^{\alpha+2} C T}\left[e^{-s t_{1}}-e^{-s t_{2}}\right]+\frac{v_{C P E, 0}}{s}
\end{aligned}
$$

Finally, using the inverse Laplace transform (3), the timedomain CPE's voltage response is the following:

$$
\begin{aligned}
& v_{C P E}(t)=v_{C P E, 0}+\frac{1}{C} * \\
& {\left[\begin{array}{l}
\frac{1}{\Gamma[\alpha+1]}\left[I_{1}\left(t-t_{1}\right)^{\alpha} u\left[t-t_{1}\right]-I_{2}\left(t-t_{2}\right)^{\alpha} u\left[t-t_{2}\right]\right]+ \\
\frac{I_{2}-I_{1}}{\Gamma[\alpha+2] T}\left[\left(t-t_{1}\right)^{\alpha+1} u\left[t-t_{1}\right]-\left(t-t_{2}\right)^{\alpha+1} u\left[t-t_{2}\right]\right]
\end{array}\right]}
\end{aligned}
$$

\section{Pulse or trapezoidal train response}

Considering that the current $I(t)$ is a continuous current signal, it is decomposed into a series of pulse square wave signal of step time $T$, as shown in Fig. 5 (on the left). Therefore, defining $n \in \mathbb{N}$ the time integer index of the pulse train signal $I(t=n T)$, it is defined by the following formula:

$$
I(n T)=\sum_{i=0}^{n-1} I_{i}[u[n T-i T]-u[n T-(i+1) T]]
$$

In this case, using (8) and (12), it is possible to describe the time-discrete CPE voltage response $v_{C P E}(n T)$ by the equation:

$$
\begin{aligned}
& v_{C P E}(n T)=v_{C P E, 0}+ \\
& \frac{T^{\alpha}}{\Gamma[\alpha+1] C} \sum_{i=0}^{n-1} I_{i}\left[(n-i)^{\alpha}-(n-(i+1))^{\alpha}\right]
\end{aligned}
$$

Otherwise, the continuous current signal $I(t)$ can be decomposed into a series of trapezoidal signal of step time $T$, as shown in Fig. 5 (on the right). In this case, using (9), the time-discrete signal $I(n T)$ it is defined by:

$$
\begin{aligned}
& I(n T)= \\
& \sum_{i=0}^{n-1}\left[\begin{array}{l}
I_{i} u[n T-i T]-I_{i+1} u[n T-(i+1) T]+\frac{I_{i+1}-I_{i}}{T} * \\
*[(n T-i T) u[n T-i T]-(n T-(i+1) T) u[n T-(i+1) T]
\end{array}\right]
\end{aligned}
$$

Now, using (11) and the input signal (14), it is possible to describe the $v_{C P E}(n T)$ :

$$
\begin{aligned}
& v_{C P E}(n T)=v_{C P E, 0}+\frac{T^{\alpha}}{C} * \\
& \sum_{i=0}^{n-1}\left[\begin{array}{l}
\frac{1}{\Gamma[\alpha+1]}\left(I_{i}(n-i)^{\alpha}-I_{i+1}(n-(i+1))^{\alpha}\right)+ \\
\frac{I_{i+1}-I_{i}}{\Gamma[\alpha+2]}\left((n-i)^{\alpha}-(n-(i+1))^{\alpha}\right)
\end{array}\right]
\end{aligned}
$$

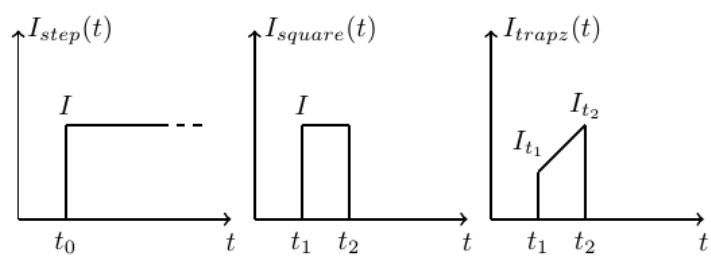

Fig. 4. Different excitation input current signal.
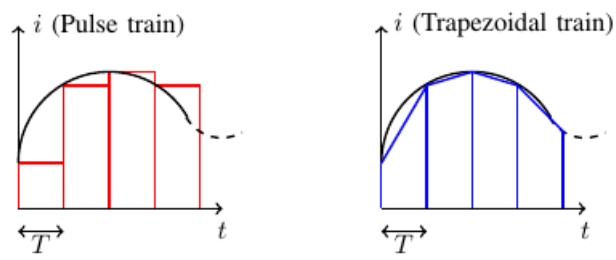

Fig. 5. Different methods for decomposition of time-continuous signals. 


\section{ANALYSIS AND MODELING OR RESISTANCE-CPE BRANCH}

\section{A. Pulse or trapezoidal response}

The components of battery EIS shown in Fig. 3 are represented by series of parallel resistance-CPEs (R-CPE) groups. In this section, the voltage response of a single group is described, by which we can describe the complete battery voltage response using the superposition principle property of linear systems. The single R-CPE group is shown in Fig. 1 (on the left).

The current passing on the $k$-th $\mathrm{CPE}$ is the following:

$$
I_{C P E_{k}}(n T)=I_{R_{0}}-\frac{v_{C P E_{k}}(n T)}{R_{k}}
$$

Based on (16), the CPE's voltage response evolution by the pulse train current signal (13) is represented by the following equation:

$$
\begin{aligned}
& v_{C P E_{k}}(n T)=v_{C P E_{k}, 0}+\frac{T^{\alpha_{k}}}{\Gamma\left[\alpha_{k}+1\right] C_{k}} * \\
& * \sum_{i=0}^{n-1}\left[\left(I_{R_{0}}(i T)-\frac{v_{C P E_{k}}(i T)}{R_{k}}\right)\left((n-i)^{\alpha_{k}}-(n-(i+1))^{\alpha_{k}}\right)\right]
\end{aligned}
$$

Finally, the CPE voltage response by the trapezoidal train current signal (15) is represented by the following equation:

$$
\begin{aligned}
& v_{C P E_{k}}(n T)=v_{C P E_{k}, 0}+\frac{T^{\alpha_{k}}}{C_{k}} * \\
& \sum_{i=0}^{n-1}\left[\begin{array}{l}
\frac{1}{\Gamma\left[\alpha_{k}+1\right]}\left[\begin{array}{l}
\left(I_{R_{0}}(i T)-\frac{v_{C P E_{k}}(i T)}{R_{k}}\right)(n-i)^{\alpha_{k}} \\
-\left(I_{R_{0}}((i+1) T)-\frac{v_{C P E_{k}}(i T)}{R_{k}}\right)(n-(i+1))^{\alpha_{k}}
\end{array}\right]+ \\
\frac{I_{R_{0}}((i+1) T)-I_{R_{0}}(i T)}{\Gamma\left[\alpha_{k}+2\right]}\left(\begin{array}{l}
(n-i)^{\left(\alpha_{k}+1\right)}- \\
-(n-(i+1))^{\left(\alpha_{k}+1\right)}
\end{array}\right)
\end{array}\right]
\end{aligned}
$$

\section{B. Stability of the system}

The single R-CPE branch model is obtained and described in the Equations (17)-(18). These are time-continuous signal models sampled with step time $T$, using respectively pulse or trapezoidal train signals. In order to study the stability of the proposed models, a second-order discrete transfer function derived from (17) and (18) is used [16]. Therefore, calculating by (17) and (18) the CPE's voltage response for the steps $i=0,1,2$, the poles of the time-discrete transfer function in the $z$ domain are equals in the case of input pulse or trapezoidal train signals [16]:

$$
\begin{gathered}
z_{\text {poles }, C P E_{k}}=\frac{-B_{k} \pm \sqrt{B_{k}^{2}-4 B_{k}\left(2^{\alpha_{k}}-1\right)}}{2} \\
B_{k}=\frac{T^{\alpha_{k}}}{\Gamma\left[\alpha_{k}+1\right] R_{k} C_{k}}
\end{gathered}
$$

It's noticeable by (19) and (20) that the stability of the model depends not only on the R-CPE values, also on the sample time $T$, therefore, on the downsampling (decimation) of the data acquired and calculated by the model. Indeed, the number of algebrical calculations in the time horizon $\left[t_{i}, t_{f}\right]$, is equal to:

$$
\begin{gathered}
7 \frac{N(N+1)}{2}+2<7 N^{2}, N>1 \\
N=\frac{t_{f}-t_{i}}{T}
\end{gathered}
$$

By (17) for pulse train signal, and:

$$
24 \frac{N(N+1)}{2}+2<24 N^{2}, N>1
$$

By (18) for trapezoidal train signal.

This result implies that the sample time $T$ of the model should be appropriately chosen in order to solve the hard trade-off between the calculation time and the model accuracy in the simulation.

\section{RESULTS}

\section{A. Experimental setup}

The evaluation of cell model parameters is performed on a LiFePO4 cell from A123, the ANR26650M1-B (2.5 Ah of capacity and $3.3 \mathrm{~V}$ of nominal voltage). The cell model parameters are estimated fitting the EIS data measurement in the frequency range [1-10] $\mathrm{Hz}$, using different Equivalent Electrical Circuit (EEC) configurations shown in Table I and in Fig. 7. It's noticeable that the fitting result's effectiveness of the all EEC configurations considered is validated. In particular, it's noticeable that in the frequency range [1-10] $\mathrm{Hz}$ we can consider only a single R-CPE branch to build the

\begin{tabular}{|c|c|c|}
\hline $\begin{array}{c}\text { EEC Model } \\
\text { Configuration }\end{array}$ & Circuit & $\begin{array}{c}\text { Estimated } \\
\text { Values }\end{array}$ \\
\hline$R_{0}-\left(R_{1} / / C P E_{1}\right)$ & $\begin{array}{c}C P E_{1} \\
R_{0} \\
\sim\end{array}$ & $\begin{array}{l}R_{0}=45.6 \mathrm{~m} \Omega \\
R_{1}=2.2 \mathrm{~m} \Omega \\
C P E_{1}= \\
{[92.53 \mathrm{~F}, 0.892]}\end{array}$ \\
\hline$L-R_{0}-\left(R_{1} / / C P E_{1}\right)$ & $\stackrel{c}{L} \quad R_{0}$ & $\begin{array}{l}L=158.7 \mathrm{nH} \\
R_{0}=45.6 \mathrm{~m} \Omega \\
R_{1}=2.2 \mathrm{~m} \Omega \\
C P E_{1}= \\
{[92.79 \mathrm{~F}, 0.883]}\end{array}$ \\
\hline $\begin{array}{c}L-R_{0}-\left(R_{1} / / C P E_{1}\right) \\
-\left(R_{2} / / C P E_{2}\right)\end{array}$ & $\begin{array}{c}C P E_{1} \\
R_{0} \\
\end{array}$ & $\begin{array}{l}R_{0}=45.6 \mathrm{~m} \Omega \\
R_{1}=1.9 \mathrm{~m} \Omega \\
C P E_{1}= \\
{[109.53 \mathrm{~F}, 0.90]} \\
R_{2}=0.4 \mathrm{~m} \Omega \\
C P E_{2}= \\
{[678.5 \mathrm{~F}, 0.80]}\end{array}$ \\
\hline
\end{tabular}
cell model. The electronic load MM540 by Material Mates Instruments has been used to spectroscopy test. The instrument has a dedicated frequency response analyzer that produces the excitation signals. The instrument is connected to a personal computer (PC) through USB protocol. The PC drives the instrument by means of a dedicated software and simultaneously collects the measurements and calculates the EIS (Fig. 6).

TABLE I. EEC CELL MODEL PARAMETERS AT SOC $70 \%$ 


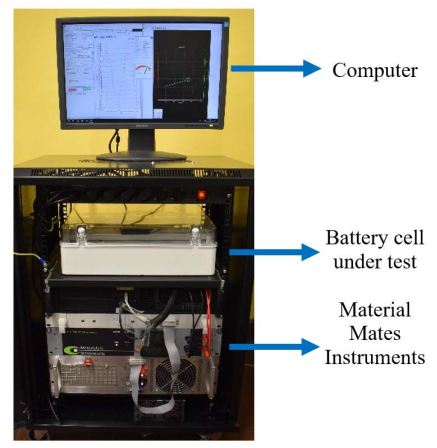

Fig. 6. Laboratory EIS test setup.

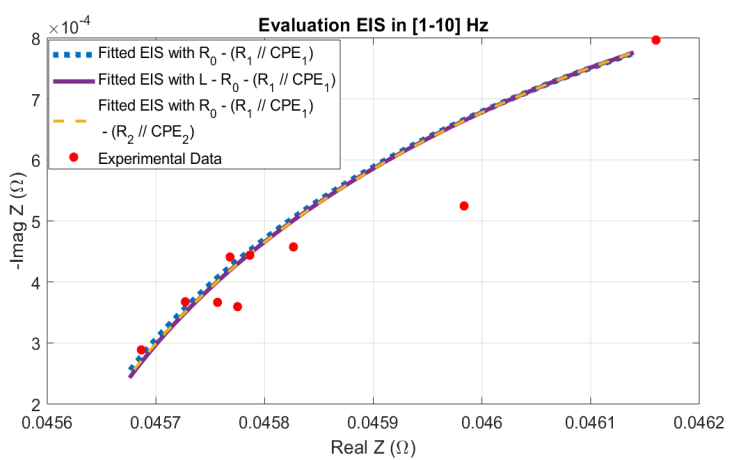

Fig. 7. Experimental EIS measurement in $[1,10] \mathrm{Hz}$ frequency range and fitted EIS curve using EEC models shown in Table I.

\section{B. Evaluation of the model}

The evaluation of EEC cell model voltage response, considering the CPE models, and the comparison between experimental results and simulation results have been evaluated. Performance of the CPE model voltage response using the pulse train method (17) and trapezoidal train method (18) are compared. In this work, the $R_{0}-\left(R_{1} / /\right.$ $\left.C P E_{1}\right)$ configuration shown in Table I is used. The Matlab software has been used to implement the CPE model, while the estimation of the voltage response evolution has been performed through acquisition of current and voltage from the selected test shown in Fig. 8 and Fig. 9, with the sample time $T=10 \mathrm{~ms}$. As mentioned before, the sample time of the model should be assessed in order to ensure its stability and good runtime during the simulation speed. Considering the original sample time $T=10 \mathrm{~ms}$, the downsampling index $\mathrm{K}$ is introduced in the EEC cell model, in order to evaluate its accuracy in the evaluation of voltage response in the step time $T_{o v}=\mathrm{K} T$. As shown in Fig. 9, the EEC model's voltage response is equal to the experimental data if there isn't downsampling in the model (so $K=1$ ). The simulation relative error is then calculated:

$$
e_{\%}(n T)=\frac{\left|v_{\text {measured }}(n T)-v_{\text {mod } e l}(n T)\right|^{2}}{\left|v_{\text {measured }}(n T)\right|^{2}} * 100
$$

Fig. 10 shows that the simulation relative error is under to the $0.5 \%$ during the test, using pulse (17) or trapezoidal (18) train method. However, considering the downsampling index $\mathrm{K}=32\left(T_{o v}=320 \mathrm{~ms}\right)$, EEC model's voltage response diverges, as shown in Fig. 9. Therefore, it is important to understand how the poles of the CPE model are moving respect to the downsampling index, considering $T=10 \mathrm{~ms}$. Fig. 11 (left) shows the root locus of the CPE model's poles. As mentioned in the previous section, the poles (19) are the same using pulse or trapezoidal methods. Analysis of the poles is conducted at different downsampling index $\mathrm{K}$ and results are shown in Fig. 11.

Time-discrete model is considered BIBO-stable if the magnitude of the poles is less than 1 . Considering the $R_{0}-$ $\left(R_{1} / / C P E_{1}\right)$ model shown in Table I, the estimation should be not conducted with $\mathrm{K} \geq 10$, as shown in Fig. 11 (left). Despite the poles of the model are the same between two methods, a further evaluation of the Root Mean Square Error (RMSE) is conducted at different downsampling index $\mathrm{K}$. RMSE is calculated by the following equation, using (24):

$$
R M S E=\sqrt{\frac{1}{N} \sum_{n=0}^{N} e_{\%}\left(\mathrm{nT}_{o v}\right)}
$$

Results are shown in Fig. 11 (right) and prove that the EEC model accuracy using the trapezoidal train method is slightly better than the pulse train method. However, as demonstrated by (23) and (21), trapezoidal train method requires more algebraic operations.

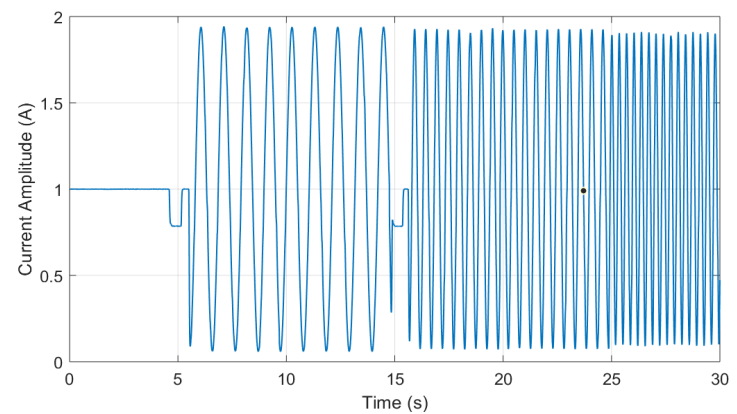

Fig. 8. Input current profile performed by EIS test setup (swept sine, 1 to $10 \mathrm{~Hz})$.

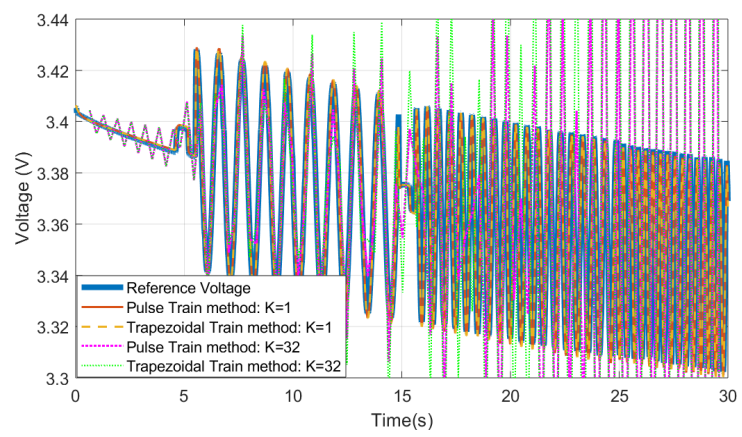

Fig. 9. Comparison between experimental and EEC simulation model $\left(R_{0}-\right.$ $\left.\left(R_{1} / / C P E_{1}\right)\right)$ battery voltage response (with $T=10 \mathrm{~ms}$ ).

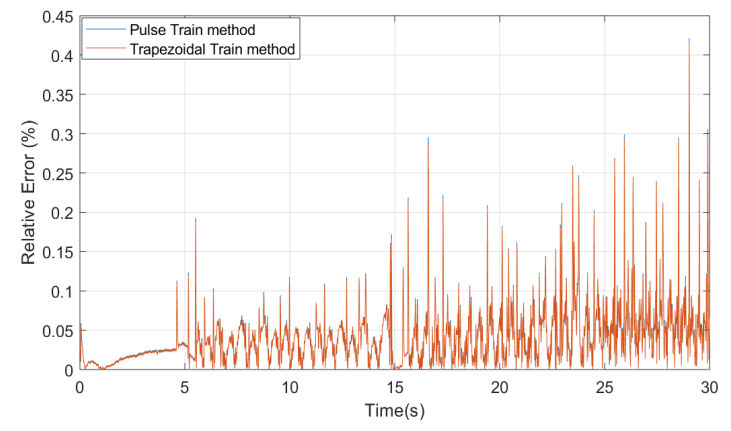

Fig. 10. Simulation relative error compare with experimental results $(K=1$ and $T=10 \mathrm{~ms}$ ). 

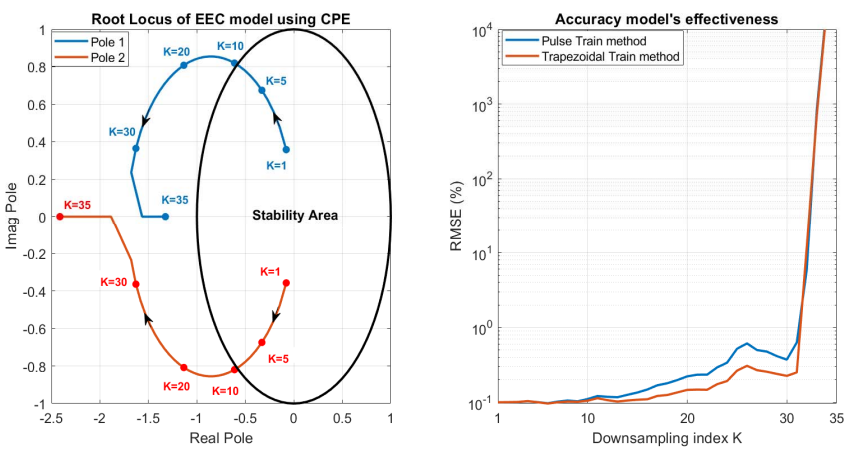

Fig. 11. Poles values (left) and simulation RMSE obtained (right) at different downsampling index $K$.

\section{CONCLUSIONS}

This paper presents two different methodologies to define a time-domain EEC cell model using the CPE, which are most important for EIS analysis. The effectiveness of these models is evaluated by the comparison between experimental data acquired by a LiFePO4 cell and simulation data, using the two different CPEs model: pulse train method or trapezoidal train method. Detailed analysis of stability and the step size of the model solver has been conducted. The proposed two models give a higher accuracy in voltage response prediction, despite a high calculation time.

\section{REFERENCES}

[1] O. Rahbari, N. Omar, Y. Firouz, M.A. Rosen, S. Goutam, P. Van Den Bossche, J. Van Mierlo: "A novel state of charge and capacity estimation technique for electric vehicles connected to a smart grid based on inverse theory and a metaheuristic algorithm", Energy 2018, 155, 1047-1058.

[2] T. Huria, G. Ludovici, G. Lutzemberger, "State of charge estimation of high power lithium iron phosphate cells", Journal of Power Sources, Volume 249, Pages 92-102, 1 March 2014.

[3] E. Locorotondo, L. Pugi, L. Berzi, M. Pierini and A. Pretto, "Online State of Health Estimation of Lithium-Ion Batteries Based on Improved Ampere-Count Method," 2018 IEEE International Conference on Environment and Electrical Engineering and 2018 IEEE Industrial and Commercial Power Systems Europe (EEEIC / I\&CPS Europe), Palermo, 2018, pp. 1-6.

[4] D. Cittanti, A. Ferraris, A. Airale, S. Fiorot, S. Scavuzzo, and M. Carello, "Modeling Li-ion batteries for automotive application: A trade-off between accuracy and complexity," International Conference of Electrical and Electronic Technologies for Automotive, Torino 15-16 June 2017, pp.8,2017.

[5] Lin X., Stefanopoulous A. G., Perez H. E., Siegel J.B., Li Y., Anderson R. D., "Quadruple adaptive observer of the core temperature in cylindrical Li-ion batteries and their health monitoring”, in 2012 American Control Conference, ACC 2012 (pp. 578-583).

[6] Pugi, L., Favilli, T., Berzi, L., Locorotondo, E., \& Pierini, M. (2018, September). Brake Blending and Optimal Torque Allocation Strategies for Innovative Electric Powertrains. In International Conference on Applications in Electronics Pervading Industry, Environment and Society (pp. 477-483). Springer, Cham.

[7] W. Waag, C. Fleischer, and D. U. Sauer, "Critical review of the methods for monitoring of lithium-ion batteries in electric and hybrid vehicles." Journal of Power Sources 258 (2014): 321-339.

[8] M. J. B. Haverkort, "Which battery model to use?" IET Software, no. 3, pp. 445-457, 2009.

[9] L.H.J. Raijmakers, D.L. Danilov, J.P.M. van Lammeren, M.J.G. Lammers, P.H.L. Notten, "Sensorless battery temperature measurements based on electrochemical impedance spectroscopy," Journal of Power Sources, vol. 247, pp. 539-544, 2014.

[10] R. Curtain and K. Morris. "Transfer functions of distributed parameter systems: A tutorial", Automatica, 45:1101 - 1116, 2009.

[11] R. Al Nazer, V. Cattin, P. Granjon, M. Montaru, "A new optimization algorithm for a li-ion battery equivalent electric circuit identification", 9th International Conference on Modeling, Optimization.

[12] R. Al Nazer, V. Cattin, P. Granjon, M. Montaru and M. Ranieri, "Broadband Identification of Battery Electrical Impedance for HEVs," in IEEE Transactions on Vehicular Technology, vol. 62, no. 7, pp. 2896-2905, Sept. 2013.

[13] C. S. Cheng, H. S. H. Chung and R. W. H. Lau, "Time-domain modeling of constant phase element for simulation of lithium batteries under arbitrary charging and discharging current profiles," 2017 IEEE Applied Power Electronics Conference and Exposition (APEC), Tampa, FL, 2017, pp. 985-992.

[14] Y. Olofsson, J. Groot, T. Katrašnik, e G. Tavcar, "Impedance spectroscopy characterisation of automotive NMC/graphite Li-ion cells aged with realistic PHEV load profile", in Electric Vehicle Conference (IEVC), 2014 IEEE International, pp. 1-6, 2014.

[15] Berthier, F., Diard, J. P., Michel, R. "Distinguishability of equivalent circuits containing CPEs: Part I. Theoretical part." Journal of Electroanalytical Chemistry, 510(1-2), 1-11, 2001.

[16] C. S Cheng, H. S. H. Chung, R. W. H. Lau, \& K. Y. Hong(2018). Time-Domain Modeling of Constant Phase Elements for Simulation of Lithium Battery Behavior. IEEE Transactions on Power Electronics. 